Case Report

\title{
Idiopathic Juvenile Osteoporosis Presenting as Loss of Height
}

\author{
Vittoria Bucknall ${ }^{1}$, Emma C. Bhatti ${ }^{2}$, Alastair W. Murray ${ }^{1}$, Athanasios I. Tsirikos ${ }^{3}$
}

${ }^{1}$ Department of Paediatric Trauma and Orthopaedic Surgery, Royal Hospital for Sick Children, Edinburgh, UK, ${ }^{2}$ University of Edinburgh Medical School, Edinburgh, UK, ${ }^{3}$ Scottish National Spine Deformity Centre, Royal Hospital for Sick Children, Edinburgh, UK

Correspondence: atsirikos@hotmail.com

Tel.: + 447966056372

Received: December 10, 2018

Accepted: February 19, 2019

Key Words: Idiopathic Juvenile Osteoporosis - Kyphosis • Bisphosphonate Therapy - Prognosis.
Objective - To report a rare case of Idiopathic Juvenile Osteoporosis (IJO) presenting as kyphosis and loss of height. Case presentation - A 9-year-old girl presented with a 6-month history of progressive loss of height, increasing roundness of the shoulders and atraumatic mild thoracolumbar discomfort. A diagnosis of IJO was subsequently made. In the course of treatment, bisphosphonates were commenced and multiple procedures were required to address lower limb fractures and angular deformities. At 10 years follow-up at skeletal maturity and after pamidronate therapy, spontaneous resolution of spinal deformity was observed resulting in a normal range of daily and sporting activities. Conclusion - IJO is a rare condition that should be considered in paediatric patients presenting with unexplained progressive loss of height and an increasing kyphosis where other causes have been excluded.

\section{Introduction}

Idiopathic Juvenile Osteoporosis (IJO) is a rare sporadic condition characterised by low bone mass that affects the pre-pubertal paediatric population, usually between 8 and 14 years $(1,2)$. The manifestations of this disease include bone pain, dental abnormalities, short stature and skeletal fractures with subsequent deformities $(3,4)$. Although osteoporosis resolves at skeletal maturity, the deformities that develop during the acute phase of the condition affecting the spine and appendicular skeleton can have profound effects and produce long-term disability if not recognised and treated early. The clinical presentation of IJO has wide variation, thus making diagnosis challenging. We report a patient with IJO presenting primarily as progressive loss of trunk height in an otherwise healthy 9-year-old girl.

\section{Case Presentation}

A 9-year-old girl presented to clinic following referral from primary care with a 6-month history of progressive loss of height, increasing roundness of the shoulders and atraumatic mild thoracolumbar discomfort on playing sport, which was relieved by rest. The family's main concern for presentation to the General Practitioner was that they had noticed their daughter to be losing height over the preceding 6 months. The patient was otherwise fit and well without significant medical, perinatal or family history. 
Table 1. Changes in Bone Density Over the 9 Years of Serial Measurements During Follow-Up

\begin{tabular}{lllllll}
\hline Age (years) & Height $(\mathrm{cm})$ & Weight $(\mathrm{kg})$ & BMD Spine & Z Score Spine & Z Score Hip & Z Score Total \\
\hline 9 & 136.5 & 32.1 & 0.301 & -4.1 & -1.6 & -1.9 \\
10 & 140.2 & 43.1 & 0.550 & -1.2 & -1.0 & -1.6 \\
11 & 151.0 & 44.7 & 0.713 & -0.1 & -1.1 & -1.2 \\
12 & 152.5 & 46.4 & 0.797 & 0.0 & -2.2 & -1.3 \\
13 & 157.2 & 55.6 & 0.839 & -0.6 & -2.3 & -2.7 \\
14 & 157.5 & 54.5 & 0.823 & -1.2 & -2.6 & -3.1 \\
15 & 157.5 & 54.5 & 0.834 & -1.3 & N/A & -3.2 \\
16 & 157.0 & 50.9 & 0.852 & -1.4 & N/A & -3.3 \\
18 & 157.0 & 56.2 & 0.798 & -2.2 & N/A & N/A \\
\hline
\end{tabular}

*Results Not Available.

On examination, the patient was 136.5 $\mathrm{cm}$ tall with sitting height $69.5 \mathrm{~cm}$ and weight $32.1 \mathrm{~kg}$ (Table 1). The patient had an increased smooth thoracic kyphosis that was non-tender on palpation. Range of motion of the spine was full and pain free. There were no cutaneous abnormalities overlying the spine and no neurological abnormalities were identified. The patient's shoulders and pelvis were symmetrical and there was no leg length inequality. Her gait was normal throughout each phase of the gait cycle.

Haematological and biochemical parameters including serum calcium, phosphate, alkaline phosphatase, thyroid and parathyroid hormones were within normal limits. The only biochemical abnormality detected was low 25$\mathrm{OH}$ Vitamin-D of $5 \mathrm{nmol} / \mathrm{L}$ (normal range: 25-170 nmol/L). On radiographic assessment of the spine, spontaneous anterior wedge compression fractures extending from T4-T11 and across L1/L2 were noted. The vertebral endplates were biconcave with reciprocal increase in intervertebral body space. The vertebral bodies appeared osteopenic. Thoracic kyphosis and lumbar lordosis measured $60^{\circ}$ and $75^{\circ}$ respectively with negative global sagittal balance of the spine measuring $-2.5 \mathrm{~cm}$ at presentation (Fig. 1a). Further investigations were performed which included a bone marrow aspirate and bone trephine which showed no abnormalities. A Dual Energy X-ray Absorptiometry (DEXA) scan was undertaken which showed the bone mineral density (BMD) of the spine to be 0.301 and a $Z$-score of -4.1 (Table 1), figures indicative of osteoporosis. In the absence of any other attributable cause, the diagnosis of IJO was made.

The patient was commenced on bisphosphonate therapy (Pamidronate $1 \mathrm{mg} / \mathrm{kg}$ a month for 12 months followed by half dose for additional 6 months). Calcium and $\mathrm{Vi}$ tamin-D supplementation was also provided. One year following diagnosis, the BMD of the spine had increased by $82.8 \%$ and the $\mathrm{Z}$ score was -1.2 (Table 1). Repeat spinal radiographs showed improvement in the appearance of the affected vertebral bodies with gradual reconstitution of anterior vertebral height in addition to cyclical Pamidronate 'metaphyseal lines' (Fig. 1b). Thoracic kyphosis improved from $60^{\circ}$ to $30^{\circ}$ and the patient's sitting height increased by $6 \mathrm{~cm}$.

Unfortunately, the patient went onto sustain a number of appendicular skeletal fractures, each of which were sustained during normal everyday activities (Table 2). Ten months following diagnosis, she complained of atraumatic bilateral proximal tibial pain that was worse on exercise. Clinical examination revealed no obvious deformity or abnormality except for pain on palpation around 

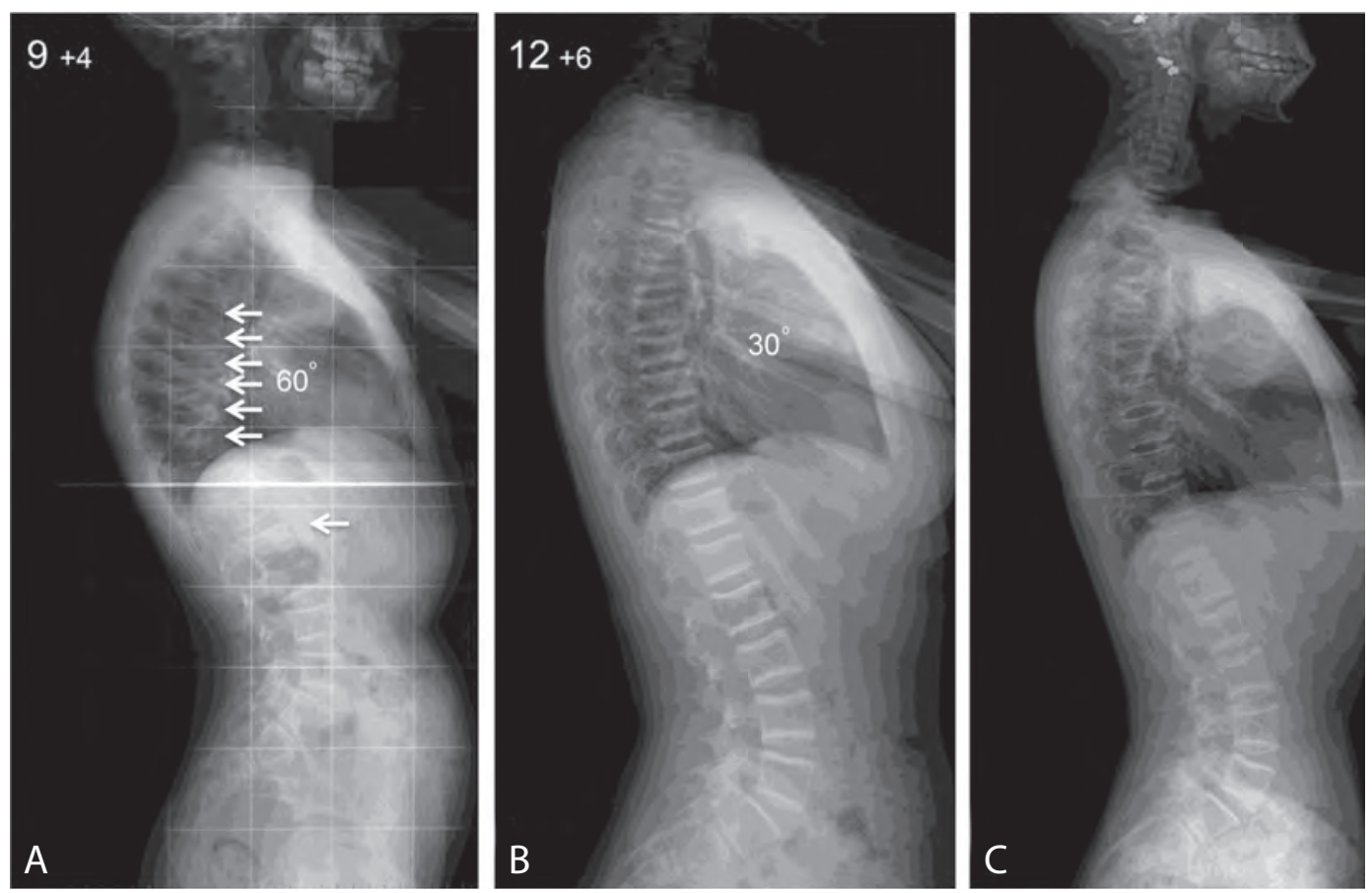

Fig. 1. Lateral Spinal Radiographs Taken at: A) Presentation Showing Compression Fractures of T4-T11 And L1/L2 Resulting in Increased Kyphosis Of $60^{\circ}$; B) 1 Year Post-Presentation Showing Improvement to $30^{\circ}$ of Kyphosis And Cyclical Pamidronate Metaphyseal Lines; C) 7 Years Post-Presentation with Maintenance of Thoracic Kyphosis and Normal Global Sagittal Balance of the Spine (Arrows Indicate Stress Fractures).

the proximal tibial metaphyseal regions bilaterally. Radiographs showed proximal tibial metaphyseal stress fractures, which were treated conservatively for 12 months with activity modification and cessation of Pamidronate treatment in the latter 6 months to encourage union. Subsequent bilateral ante- rior epiphysiodesis was undertaken to address bilateral posterior slope deformity $\left(35^{\circ}\right.$ on the left and $30^{\circ}$ on the right) using a single 4 $\mathrm{mm}$ partially threaded cancellous screw (Fig. 2a). An improvement of $15^{\circ}$ was achieved bilaterally prior to physeal closure (Fig. 2b). A multidisciplinary team decision was made

Table 2. Appendicular Skeletal Fractures Sustained by Our Patient

\begin{tabular}{lll}
\hline Age (years) & Fracture & Treatment \\
\hline 9 & Buckle fracture right distal radius. & Conservative treatment in cast - 4 weeks, \\
10 & $\begin{array}{l}\text { Bilateral proximal tibial metaphyseal stress } \\
\text { fractures. }\end{array}$ & $\begin{array}{l}\text { Conservative treatment with activity modification and delayed } \\
\text { epiphysiodesis with a single partially threaded cancellous screw. }\end{array}$ \\
11 & Left distal third femoral shaft fracture. & Operative treatment with external-fixator - 8 weeks. \\
11 & Right distal third radius fracture. & Conservative treatment in cast -4 weeks. \\
11 & Right trans-cervical femoral neck fracture. & $\begin{array}{l}\text { Operative treatment Pauwell's valgus osteotomy with dynamic } \\
\text { hip screw fixation right hip. }\end{array}$ \\
15 & Left trans-cervical femoral neck fracture. & Operative treatment with partially threaded cannulated screws. \\
17 & Left sub-trochanteric femoral fracture. & $\begin{array}{l}\text { Operative treatment with osteotomy and cephalomedullary } \\
\text { femoral nail. }\end{array}$ \\
& & Conservative treatment with analgesia. \\
\hline
\end{tabular}



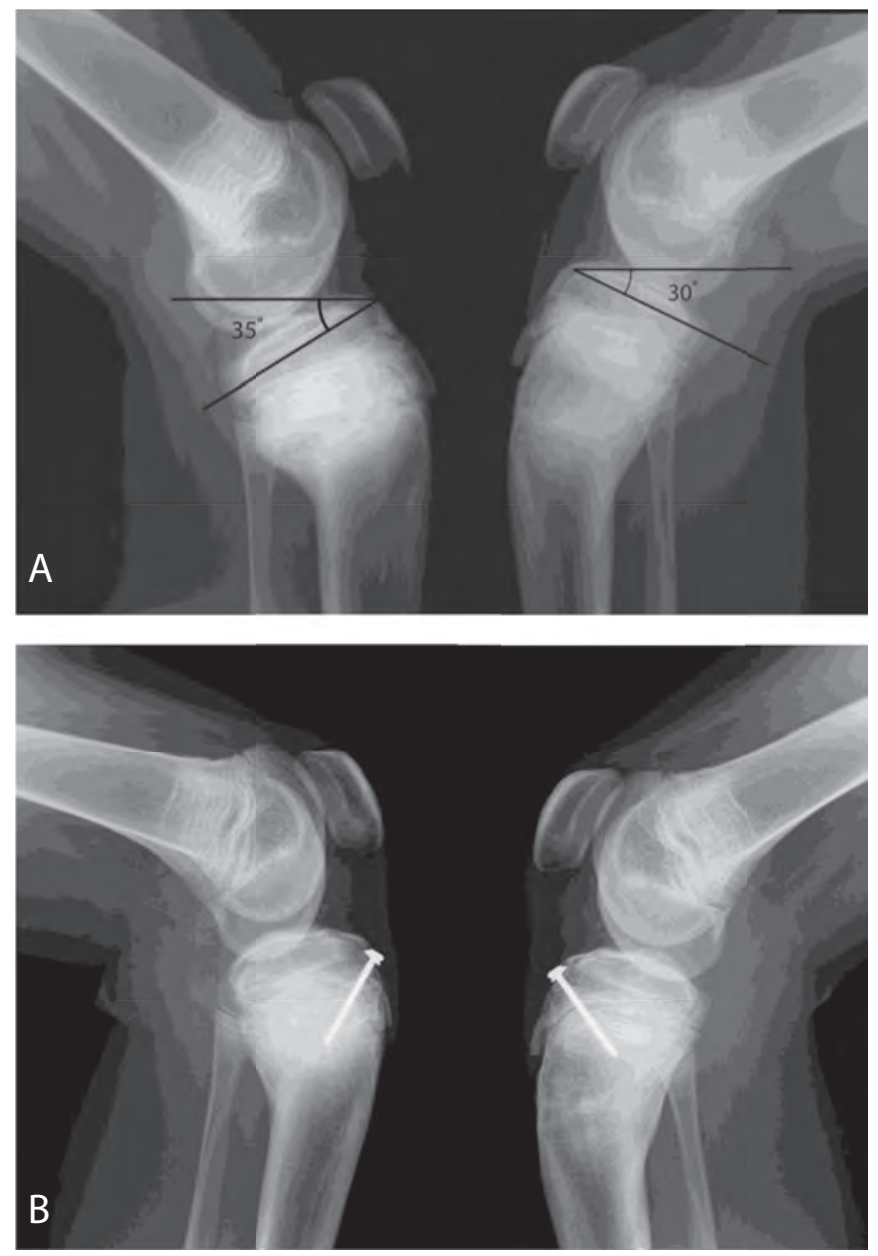

Fig. 2. Lateral Radiographs of Both Left and Right Knees Showing: A) Bilateral Proximal Tibial Stress Fractures with a Left $35^{\circ}$ and Right $30^{\circ}$ Posterior Slope Respectively; B) $15^{\circ}$ Improvement Following Epiphysiodesis Using a $4 \mathrm{~mm}$ Partially Threaded Cancellous Screw.

not to restart Pamidronate at this time due to significant improvement in BMD and Zscores on DEXA scan.

However, due to a subsequent left distal femoral fracture at age 11 years treated with external fixation, Pamidronate was recommenced at a dose of $1 \mathrm{mg} / \mathrm{kg}$ for 2 days every 2 months for 1 year followed by $1 \mathrm{mg} / \mathrm{kg}$ for 2 days every 4 months continued. Despite this, the patient complained of right hip discomfort only 2 months following removal of her left femoral external fixator. Radiographs showed a right femoral neck stress fracture requiring operative treatment, which in- cluded a Pauwell's valgus osteotomy (Fig. 3). Four years later, the patient described similar discomfort in her left hip. Radiological investigations including MRI scanning indicated a left trans-cervical femoral neck stress fracture. Cannulated screw fixation of the left hip was subsequently undertaken at age 15 years.

Two years following her left hip fixation, the patient again started complaining of left hip pain. Radiographs revealed a subtrochanteric stress fracture. This necessitated removal of the cannulated screws and insertion of a cepho-medullary nail. The fracture subsequently went onto union. 

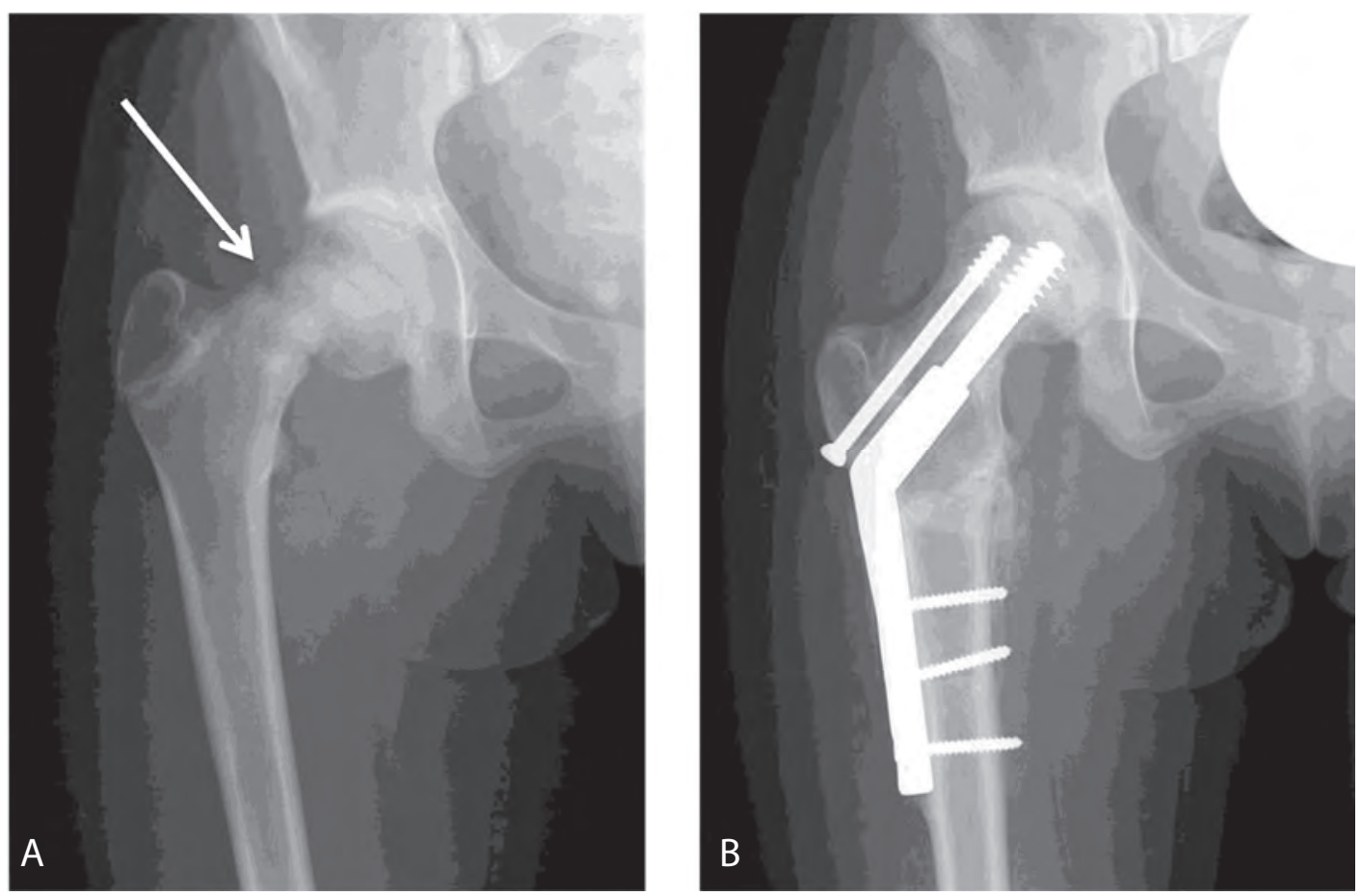

Fig. 3. AP Radiograph of the Right Hip Showing. A) an Intra-Capsular Stress Fracture of the Right Femoral Neck B) Union Following Operative Fixation with Pauwell's Valgus Osteotomy (Arrows Indicate Stress Fracture).

At age 16 years, the patient had no complaints of her back and had resumed normal activities including sports. BMD of her spine was $0.852, \mathrm{Z}$-score -1.4 and thoracic kyphosis had improved from the initial $60^{\circ}$ to $36^{\circ}$ (Fig. 1c). Lumbar lordosis had improved from initial $75^{\circ}$ to $50^{\circ}$ and global sagittal balance from initial $-2.5 \mathrm{~cm}$ to $-0.8 \mathrm{~cm}$. Furthermore, Vitamin D levels had returned to within normal limits. On clinical examination, there was no cosmetic deformity and the spine was well-balanced with normal range of pain free movement. Pamidronate therapy was stopped.

Ten years following diagnosis, the patient is leading an active lifestyle, regularly cycling and swimming. However, higher level sporting activity is restricted by the onset of lumbar discomfort which is managed conservatively. With regards to her spine, hips and knees, she maintains a full range of pain free movement at rest. Radiographs show the femoral osteotomy to have united and there is no current evidence of implant loosening, fatigue or avascular necrosis of the femoral heads. She has achieved a functional outcome and has been discharged from routine clinical care.

\section{Discussion}

IJO can produce significant patient morbidity at a stage of rapid skeletal growth and high level of activities. There is a paucity of information within the current literature limited to approximately 100 case reports $(2,3)$. This rare masquerading condition presents a diagnostic conundrum for physicians and surgeons.

IJO is a condition affecting primary bone mineralisation characterised by reduced bone mass and structural integrity, as well as increased risk of fractures $(5,6,7)$. It is a diagnosis of exclusion when other primary and 
secondary causes of osteoporosis (BMD $<-2$ standard deviations/Z score of -2 or below) have been fully investigated and excluded $(4,7,8)$. The list of differential diagnoses is extensive but each must be carefully considered. Primary causes of bone demineralisation include osteogenesis imperfecta, which results in a structural defect in Type I collagen. Osteogenesis imperfecta type I, II and III may be easily differentiated by the absence of scleral involvement, hearing deficits and their severe progressive nature. Type IV and the newer types V, VI and VII can be excluded as it was in this case by the absence of family history due to the autosomal dominant pattern of inheritance $(9,10)$. There has been so far no conclusive evidence of inheritable genetic mutations associated with IJO (11).

Secondary causes of bone demineralisation include rickets, which is characterised by abnormal values of alkaline phosphatase, phosphate, serum calcium and pathognomonic radiological changes. Although our patient had a low Vitamin D level, each of the other biochemical parameters which could suggest rickets were within normal limits. Metabolic causes of osteoporosis require investigations and are associated with abnormal levels of serum urea, creatinine, urine $\mathrm{pH}$ and blood gases. Endocrinopathies necessitate hormonal assays and Wilson's disease is characteristically identified by abnormal ceruloplasmin and copper values $(3,8)$.

Clinical presentation along with biochemical, histopathological and radiological investigations substantiated the diagnosis of IJO in our patient. The most frequently described presenting features of IJO are inability to mobilise, multiple appendicular skeletal fractures and back pain, the latter being a mild presenting feature in our patient (12). However, it was the progressive loss of trunk height prior to any long bone fractures that was the trigger for referral in our patient. The natural course of IJO is self-limiting with remission occurring after puberty. Current treatment includes activity modification to limit the occurrence of fragility fractures during the active phase of skeletal growth. In addition, recognised medical therapies include bisphosphonate treatment, calcium and Vitamin - D supplementation to assist bone formation and mineralisation; all these therapies were used in the case described (3).

\section{Conclusion}

The presentation of loss of trunk height combined with increased kyphosis and pain in an otherwise healthy child should raise clinical suspicion of a disorder of bone demineralisation with IJO featuring as a differential diagnosis.

\section{Learning Points:}

- Idiopathic Juvenile Osteoporosis (IJO) is a rare 'diagnosis of exclusion' affecting primary bone mineralisation.

- IJO should be considered in paediatric patients presenting with loss of trunk height, kyphosis and fragility fractures.

- Early diagnosis and treatment aims to prevent further fractures and subsequent skeletal deformity.

- Bisphosphonate treatment has been shown to enhance vertebral body height recovery with subsequent improvement in thoracic kyphosis and global sagittal balance of the spine.

Authors' Contributions: Conception and design: AIT, VB; Acquisition, analysis and interpretation of data: AIT, VB, EB; Drafting the article: AIT, VB; Revising the article critically for intellectual content: AIT, VB, EB, AWM; Approved final version of the manuscript: AIT, VB, EB, AWM.

Conflict of Interest: The authors declare that they have no conflict of interest. 


\section{References}

1. Hendrickx G, Boudin E, Van Hul W. A Look Behind the Scenes: the risk and pathogenesis of primary osteoporosis. Nat Rev Rheumatol. 2015;11(8):462-74.

2. Garcia I, Chiodo V, Ma Y, Boskey A. Evidence of altered matrix composition in iliac crest biopsies from patients with idiopathic juvenile osteoporosis. Connective tissue research. 2016;2;57(1):28-37.

3. Imerci A, Canbek U, Haghari S, Surer L, Kocak M. Idiopathic Juvenile Osteoporosis: A case report and review of the literature Int J Surg Case Rep. 2015; 9:127-29.

4. Altan H, Tosun G, Sen Y. Idiopathic Juvenile Osteoporosis: A case report. J Clin Diagn Res. 2015;9(8):ZD10-ZD12.

5. Consensus A. Consensus Development Conference, Prophylaxis and Treatment of Osteoporosis. Am J Med. 1993;94(6):646-50.
6. Rauch F, Travers R, Norman ME. Deficient Bone Formation in Idopathic Juvenile Osteoporosis: a histomorphometric study of cancellous iliac bone. J Bone Miner Res. 2000;15:957-63.

7. Ward LM, Ma J. Osteoporosis: Diagnosis and Management. In: Radovick S, Misra M, editors. Pediatric Endocrinology. Cham: Springer; 2018. p. 525-65.

8. Kulkarni ML, Keshavamurthy KS. Juvenile Idiopathic Osteoporosis: Indian paediatrics. 2004;41:737-40.

9. Smith R. Idiopathic Osteoporosis in the Young. J Bone Joint Surg. 1980;62-B (4): 417-27.

10. Van Dijk FS, Pals G, Van Rijn RR, Nikkels PG, Cobben JM. Classification of Osteogenesis Imperfecta Revisited. Eur J Med Genet. 2010;53(1):1-5.

11. Krassas GE. Idiopathic Juvenile Osteoporosis. Ann NY Acad Sci. 2000;900:409-12.

12. Chlebna SD, Loba JE, Sikora A. Clinical Evaluation of Patients with Idiopathic Juvenile Osteoporosis. J Pediatr Orthop B. 2001;10:259-63. 